Pacific Journal of Mathematics

ON THE DENSITY OF TWISTOR ELEMENTARY STATES 


\title{
ON THE DENSITY OF TWISTOR ELEMENTARY STATES
}

\author{
M. G. Eastwood and A. M. Pilato
}

\begin{abstract}
$\mathrm{U}(p, q)$ may be represented on $H^{p-1}\left(\mathbf{P}^{+}, \mathscr{O}(\lambda)\right)$ where $\mathbf{P}^{+}$is an open orbit of $\mathrm{U}(p, q)$ in $\mathbf{C P}_{p+q-1}$ and $\lambda$ is a homogeneous holomorphic line bundle. Although it is not their definition, the twistor elementary states turn out to be the $\mathrm{U}(p) \times \mathrm{U}(q)$-finite vectors. We show that $H^{p-1}\left(\mathbf{P}^{+}, \mathscr{O}(\lambda)\right)$ has a natural Fréchet space topology in which these states are dense. Using this, we show that a certain Hermitian product defined on $H^{p-1}\left(\overline{\mathbf{P}^{+}}, \mathscr{O}(\lambda)\right)$ is positive definite and hence complete a twistor construction of a family of unitary representations of $\mathrm{U}(p, q)$, namely the ladder representations. Though these representations are well-studied by other means, we feel that their realization on cohomology is especially natural and merits special investigation.
\end{abstract}

1. Introduction. Let $\mathbf{T}$ denote a fixed complex vector space equipped with a non-degenerate Hermitian form $\Phi$ having $p$ positive eigenvalues and $q$ negative eigenvalues. Denote by $\mathbf{P}^{+}$and $\mathbf{P}^{-}$ the subsets of the projective space $\mathbf{P} \equiv \mathbf{P T}$ on which $\boldsymbol{\Phi}$ is positive or negative respectively (a homogeneous condition). Let $V$ be a $\mathrm{GL}(\mathbf{T})-$ homogeneous vector bundle on $\mathbf{P}$. Then via its action on $\mathbf{T}, \mathrm{U}(p, q)$ is represented on the cohomologies

$$
H^{r}\left(\mathbf{P}^{+}, \mathscr{O}(V)\right) \text { and } H^{r}\left(\overline{\mathbf{P}^{+}}, \mathscr{O}(V)\right) \text {. }
$$

We will consider below only the case of a homogeneous line bundle $\lambda$ though much of the analysis applies generally (cf. [16]). The study of these representations is one of the motivations for this article.

Another motivation, which also provides some of the machinery for studying these cohomologies is that of twistor theory and especially the Penrose transform, a key aspect of twistor theory. Classical twistor theory [34, 35, 36, 37], as initiated and developed by Roger Penrose, is concerned with the case $p=q=2$ and is based on the 4-1 covering $\operatorname{SU}(2,2) \rightarrow C_{+}^{\uparrow}(1,3)$ where $C_{+}^{\uparrow}(1,3)$ is the identity connected component of the group of conformal motions of conformally compactified Minkowski space (see [34]). In this case the Penrose transform [12] identifies $H^{1}\left(\mathbf{P}^{+}, \mathscr{O}(\lambda)\right)$ with solutions of certain field equations on compactified Minkowski space called the massless 
field equations. The invariance of these equations under $C_{+}^{\uparrow}(1,3)$ is well-known [33] but is clear from the twistor perspective (i.e. via the Penrose transform). The construction of an invariant Hermitian product usually called the scalar product is physically well motivated [19] but it takes some effort to show [24] its invariance. However, once the definition of scalar product has been transferred to $H^{1}\left(\overline{\mathbf{P}^{1}}, \mathscr{O}(\lambda)\right)$ (accomplished in $[35,20,21,13])$ its invariance is clear. Proving that the scalar product is positive definite using the Minkowski space definition may also be indirect depending on whether the field is "Bosonic" or "Ferminonic". The Bosonic case is usually approached via an expansion in plane waves, i.e. by means of the Fourier transform, or via an expansion in Grgin's spherical harmonics [23]. The latter become an expansion in elementary states under the Penrose transform.

Many other investigations in twistor theory have been carried out under the assumption that it is enough to consider elementary states. This has given rise to a calculus for manipulating elementary states known as the theory of twistor diagrams [35, 36, 27, 26]. The density proved in this paper goes some way towards justifying this approach.

This article is organized as follows. Section 2 describes some results on pseudoconvexity which are used to investigate $H^{r}\left(\mathbf{P}^{+}, \mathscr{O}(\lambda)\right)$. The elementary states are introduced in $\S 3$ and the density and consequences are described in $\S \S 5,6$, and 7 . As an interlude, $\S 4$ contains a review of other approaches to the unitarization of the ladder representations and a comparison with the twistor approach of this article. Whilst more details can be found in $\S 4$, we should point out here that the twistor construction is on Dolbeault cohomology and this is not the same nor easily related to realizations on $L^{2}$-cohomology (as in the work of Rawnsley, Schmid, and Wolf [38]).

The key ingredient of the twistor method is the Penrose transform. The details of this transform are omitted from this article. They can be found in $[14,15,16]$. More links between the Penrose transform and representation theory are described in [6]. Twistor theory background may be found in [36, 27, 37].

The initial version of this paper was somewhat confused, partly owing to our limited knowledge of representation theory. We thank the referee for several helpful comments in this area and for suggesting the current organization of the paper. Conversations with John Rice were also helpful in this regard.

2. Generalities on cohomologies. Throughout this section $V$ is an arbitrary complex vector bundle on $\mathbf{P}$. 
It is convenient to choose coordinates $\left(Z^{1}, Z^{2}, \ldots, Z^{n}\right)$ on $\mathbf{T}$ such that $\Phi$ takes the form

$$
\Phi(Z, Z)=\left|Z^{1}\right|^{2}+\left|Z^{2}\right|^{2}+\cdots+\left|Z^{p}\right|^{2}-\left|Z^{p+1}\right|^{2}-\cdots-\left|Z^{n}\right|^{2} .
$$

Let $\Psi$ denote the positive definite Hermitian product

$$
\Psi(Z, Z) \equiv\left|Z^{1}\right|^{2}+\left|Z^{2}\right|^{2}+\cdots+\left|Z^{n}\right|^{2}
$$

and introduce the function $\phi \equiv \Phi / \Psi$ on $\mathbf{P}$. Then one has the following picture

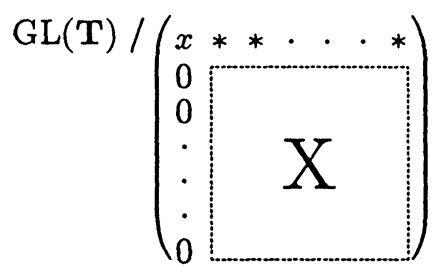

where $L=\left\{Z^{1}=Z^{2}=\cdots=Z^{p}=0\right\}$, a linearly embedded $\mathbf{P}_{q-1}$. It is easy to compute that the complex Hessian of $\phi$ has $p-1$ positive eigenvalues and $q$ negative eigenvalues on $\mathbf{P}^{+}$(related and similarly motivated functions are to be found in [40]). Thus, using $1 / \phi$ as an exhaustion function for $\mathbf{P}^{+}$, it follows from [2] (see also [25] and [3]) that

$$
H^{r}\left(\mathbf{P}^{+}, \mathscr{O}(V)\right)=0 \text { for all } r \geq p .
$$

On $\mathbf{P}^{-}$, however, $\phi$ has $p$ positive eigenvalues and $q-1$ negative eigenvalues. The results on pseudoconcavity in [2] show that

$$
H^{r}(\mathbf{P}-L, \mathscr{O}(V)) \rightarrow H^{r}\left(\mathbf{P}^{+}, \mathscr{O}(V)\right)
$$

is an isomorphism for $r<p-1$ and injective for $r=p-1$. One can now compute $H^{r}(\mathbf{P}-L, \mathscr{O}(V))$ using the Leray cover $\left\{Z^{1} \neq 0\right\}$, $\ldots,\left\{Z^{p} \neq 0\right\}$ but this is essentially the method used by Scheja [39] to prove a cohomological version of the Riemann removable singularities theorem which implies that

$$
H^{r}(\mathbf{P}-L, \mathscr{O}(V))=H^{r}(\mathbf{P}, \mathscr{O}(V))
$$

for $0 \leq r \leq \operatorname{codim} L-2$, i.e. for $0 \leq r \leq p-2$. $H^{r}(\mathbf{P}, \mathscr{O}(V))$ may be computed by Bott's theorem [7, 15] but in any case is finite dimensional [9]. For a homogeneous line bundle $\lambda$, Bott's theorem implies that $H^{r}(\mathbf{P}, \mathscr{O}(\lambda))=0$ except possibly for $r=0$ or $n-1$.

To summarize these facts:

$$
H^{r}\left(\mathbf{P}^{+}, \mathscr{O}(V)\right)
$$


vanishes for $r \geq p$. Only for $r=p-1$ is the cohomology infinite dimensional and then

$$
H^{p-1}(\mathbf{P}-L, \mathscr{O}(V)) \rightarrow H^{p-1}\left(\mathbf{P}^{+}, \mathscr{O}(V)\right)
$$

is injective. For $r \leq p-2$ one has

$$
H^{r}\left(\mathbf{P}^{+}, \mathscr{O}(V)\right)=H^{r}(\mathbf{P}, \mathscr{O}(V)) .
$$

With rather more effort these facts may be established using the Penrose transform $[\mathbf{1 5}, \mathbf{6}]$. Similar conclusions apply to $H^{r}\left(\overline{\mathbf{P}^{+}}, \mathscr{O}(V)\right)$.

For an arbitrary holomorphic vector bundle $V$ on a complex manifold $X$, one can always endow $H^{r}(X, \mathscr{O}(V))$ with a topology [45, 32] by using the topology of uniform convergence on compact sets for the Dolbeault resolution of $\mathscr{O}(V)$. However, since $\bar{\partial}$ need not have closed range (a counterexample is provided in [45]), the resulting topology on the cohomology need not be Hausdorff. If the topology is Hausdorff, then $H^{r}(X, \mathscr{O}(V))$ is a Fréchet space. When $H^{r+1}(X, \mathscr{O}(V))$ is Hausdorff, Serre duality identifies the continuous dual of $H^{r}(X, \mathscr{O}(V))$ as the compactly supported cohomology $H_{*}^{m-r}\left(X, \Omega^{m}\left(V^{*}\right)\right)$ where $m=\operatorname{dim} X$ and $\Omega^{m}$ denotes the holomorphic $m$-forms. On the other hand, Laufer [32] shows (more than) the converse: if $H^{r}(X, \mathscr{O}(V))$ has $H_{*}^{m-r}\left(X, \Omega^{m}\left(V^{*}\right)\right)$ as continuous dual then $H^{r+1}(X, \mathscr{O}(V))$ is Hausdorff. Also the topology is always Hausdorff on a finite dimensional cohomology [45].

THEOREM 1. $H^{p-1}\left(\mathbf{P}^{+}, \mathscr{O}(V)\right)$ is a Fréchet space with dual $H_{*}^{q}\left(\mathbf{P}^{+}, \mathscr{O}(\widetilde{V})\right)$ where $\widetilde{V}$ is the vector bundle $\Omega^{n-1}\left(V^{*}\right)$.

Proof. By the discussion above it is enough to show that $H_{*}^{q+1}\left(\mathbf{P}^{+}, \mathscr{O}(\widetilde{V})\right)$ is the dual of $H^{p-2}\left(\mathbf{P}^{+}, \mathscr{O}(V)\right)$. According to earlier argument, however, $H^{p-2}\left(\mathbf{P}^{+}, \mathscr{O}(V)\right)=H^{p-2}(\mathbf{P}, \mathscr{O}(V))$ whereas $H_{*}^{q+1}\left(\mathbf{P}^{+}, \mathscr{O}(\widetilde{V})\right)$ may be identified with the aid of the exact sequence [22]

$$
\begin{aligned}
H^{q}\left(\overline{\mathbf{P}^{-}}, \mathscr{O}(\tilde{V})\right) & \rightarrow H_{*}^{q+1}\left(\mathbf{P}^{+}, \mathscr{O}(\tilde{V})\right) \rightarrow H^{q+1}(\mathbf{P}, \mathscr{O}(\widetilde{V})) \\
& \rightarrow H^{q+1}\left(\overline{\mathbf{P}^{-}}, \mathscr{O}(\widetilde{V})\right) .
\end{aligned}
$$

In this sequence the first and last terms vanish by previous considerations of pseudoconvexity. Thus, we are required to prove that $H^{q+1}(\mathbf{P}, \mathscr{O}(\widetilde{V}))$ is the dual of $H^{p-2}(\mathbf{P}, \mathscr{O}(V))$. This is the usual Serre duality [45] for compact manifolds. 
Note also that $H_{*}^{q}\left(\mathbf{P}^{+}, \mathscr{O}(\widetilde{V})\right)$ may be related to $H^{q-1}\left(\overline{\mathbf{P}^{-}}, \mathscr{O}(\tilde{V})\right)$ by means of the exact sequence

$$
\begin{aligned}
H^{q-1}(\mathbf{P}, \mathscr{O}(\widetilde{V})) & \rightarrow H^{q-1}\left(\overline{\mathbf{P}^{-}}, \mathscr{O}(\tilde{V})\right) \rightarrow H_{*}^{q}\left(\mathbf{P}^{+}, \mathscr{O}(\tilde{V})\right) \\
& \rightarrow H^{q}(\mathbf{P}, \mathcal{O}(\tilde{V})) .
\end{aligned}
$$

The extreme terms are always finite dimensional so $H_{*}^{q}\left(\mathbf{P}^{+}, \mathscr{O}(\tilde{V})\right)$ is almost isomorphic to $H^{q-1}\left(\overline{\mathbf{P}^{-}}, \mathscr{O}(\widetilde{V})\right)$. In the case of a homogeneous line bundle $\lambda$, if $p \geq 2$ and $q \geq 2$, then

$$
H^{q-1}(\mathbf{P}, \mathscr{O}(\tilde{\lambda}))=0=H^{q}(\mathbf{P}, \mathscr{O}(\tilde{\lambda}))
$$

so an isomorphism is obtained. As far as constructing the ladder representations is concerned, the twistor construction when $p=1$ and $q=1$ is slightly degenerate as explained in $[\mathbf{1 4}, \mathbf{6}]$. This degeneracy is exactly reflected in the finite dimensional "corrections" as above. Thus, from now on we shall assume that $p, q \geq 2$ leaving the special cases $p=1$ or $q=1$ to the reader.

In this case Theorem 1 can be rephrased above as a duality between $H^{p-1}\left(\mathbf{P}^{+}, \mathscr{O}(\lambda)\right)$ and $H^{q-1}\left(\overline{\mathbf{P}^{-}}, \mathscr{O}(\tilde{\lambda})\right)$. One can easily check that the pairing between these cohomologies is given as follows (cf. [45]). For $\omega \in H^{q-1}\left(\overline{\mathbf{P}^{-}}, \mathscr{O}(\tilde{\lambda})\right)$ choose $U$ an open neighbourhood of $\overline{\mathbf{P}}^{-}$so that $\omega$ is represented by $\tilde{\omega} \in H^{q-1}(U, \mathscr{O}(\tilde{\lambda}))$. Now for $\mu \in$ $H^{-1}\left(\mathbf{P}^{+}, \mathscr{O}(\lambda)\right)$ form the cup product $\tilde{\omega} \cap \mu \in H^{n-2}\left(U \cap \mathbf{P}^{+}, \Omega^{n-1}\right)$ and finally take the Mayer-Vietoris connecting homomorphism for the cover $\left\{U, \mathbf{P}^{+}\right\}$of $\mathbf{P}$ to obtain an element of $H^{n-1}\left(\mathbf{P}, \Omega^{n-1}\right)=\mathbf{C}$. This is the dot product $\omega \cdot \mu$ introduced in [13].

Laufer [32] also discusses topologies on the compactly supported cohomology $H_{*}^{r}(X, \mathscr{O}(V))$ and proves that if $H_{*}^{r+1}(X, \mathscr{O}(V))$ has a Hausdorff topology, then $H^{m-r}(X, \mathscr{O}(\widetilde{V}))$ is the continuous dual of $H_{*}^{r}(X, \mathscr{O}(V))$. Using this result, the argument above for $H^{p-1}\left(\mathbf{P}^{+}, \mathscr{O}(V)\right)$ may be repeated for $H^{p-1}\left(\overline{\mathbf{P}^{+}}, \mathscr{O}(V)\right)$ to prove:

THEOREM 2. Utilizing the notation of the introduction, suppose that $p, q \geq 2$, and that $\lambda$ is a homogeneous line bundle on $\mathbf{P}$. Then $H^{q-1}\left(\overline{\mathbf{P}^{-}}, \mathscr{O}(\lambda)\right)$ has the natural topology of the strong dual of a Fréchet space and the dot product

$$
H^{p-1}\left(\mathbf{P}^{+}, \mathscr{O}(\lambda)\right) \otimes H^{q-1}\left(\overline{\mathbf{P}^{-}}, \mathscr{O}(\tilde{\lambda})\right) \rightarrow \mathbf{C}
$$

is a perfect pairing.

Note that the rôles of $\mathbf{P}^{+}$and $\mathbf{P}^{-}$above may be reversed by replacing $\Phi$ by $-\Phi$. Also note that Laufer [32] provides alternative 
methods of describing these natural topologies and, in particular, it is possible directly to use the Čech definition of cohomology. The Čech method is commonly employed in twistor theory.

3. Elementary states. The twistor elementary states are certain cohomology classes in $H^{1}(\mathbf{P}-L, \mathscr{O}(\lambda))$ as described later in this section. As a homogeneous space for $\operatorname{GL}(\mathbf{T}), \mathbf{P}$ is the quotient

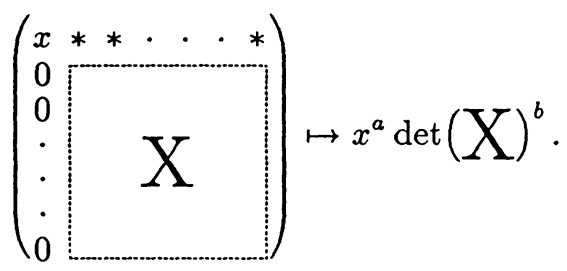

and the homogeneous line bundles are given by the representations

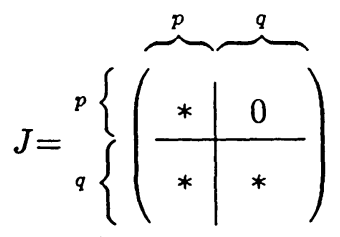

In line with the notation of [15], this line bundle will be denoted by $(a \mid b, b, \ldots, b)$. Note that $(-1 \mid 0, \ldots, 0)$ is the hyperplane section bundle: $\mathscr{O}(k)=(-k \mid 0, \ldots, 0)$. If $\operatorname{SL}(\mathbf{T})$ is used instead then only $a-b$ is relevant since

$$
(a \mid b, \ldots, b)=(a-b \mid 0, \ldots, 0) \otimes(\operatorname{det} \mathbf{T})^{b}=\mathscr{O}(b-a) \otimes(\operatorname{det} \mathbf{T})^{b} .
$$

Following Laufer [31], it is shown in [11] how $H^{p-1}(\mathbf{P}-L, \mathscr{O}(k))$ may be represented in terms of Laurent series. An element in $H^{p-1}(\mathbf{P}-L, \mathscr{O}(k))$ may be written

$$
\sum_{\alpha, \beta, \ldots, \gamma>0} \frac{A_{\alpha \beta \cdots \gamma}\left(Z^{p+1}, \cdots, Z^{n}\right)}{\left(Z^{1}\right)^{\alpha}\left(Z^{2}\right)^{\beta} \cdots\left(Z^{p}\right)^{\gamma}}
$$

where $A_{\alpha \beta \cdots \gamma}$ is a homogeneous polynomial of degree $\alpha+\beta+\cdots+\gamma+\frac{k}{\mathrm{j}}$. Each term in this expansion is included in $H^{p-1}(\mathbf{P}-L, \mathscr{O}(k))$ as a Cech representative with respect to the Leray cover $\left\{Z^{1} \neq 0\right\}, \ldots$, $\left\{Z^{p} \neq 0\right\}$ of $\mathbf{P}-L$. Evidently, $\mathrm{U}(q)$ acts on each $A^{\alpha \beta \cdots \gamma}$ according to the totally symmetric dual representation $\left(\operatorname{Sym}^{d+k}\right)^{*}$, where $d=\alpha+$ $\beta+\cdots+\gamma$. The action of $\mathrm{U}(p)$ on the variables $\left(Z^{1}, \ldots, Z^{p}\right)$ is less 
obvious as it involves changing the Čech cover but it is straightforward to verify that $d$ is preserved and that the corresponding representation on the collection

$$
\left\{\frac{1}{\left(Z^{1}\right)^{\alpha}\left(Z^{2}\right)^{\beta} \cdots\left(Z^{p}\right)^{\gamma}}\right\}_{\alpha+\beta+\cdots+\gamma=d}
$$

is the totally symmetric representation $\operatorname{Sym}^{d-p}$ tensored with the determinant representation. Overall, therefore the terms in the Laurent series expansion for each fixed $d$ provide a finite dimensional irreducible representation of $\mathrm{U}(p) \times \mathrm{U}(q)$, namely (in the notation of [15]) the representation

$$
(1, \ldots, 1,1+d-p \mid-k-d, 0, \ldots, 0) \text { for } d \geq \max (p,-k) \text {. }
$$

Finite linear combinations of these elements of $H^{p-1}(\mathbf{P}-L, \mathscr{O}(k))$ are called elementary states. In other words, the elementary states are represented by finite Laurent expansions (see [11] and [6] for further discussion). Notice that $\mathbf{P}-L$ is a homogeneous space for

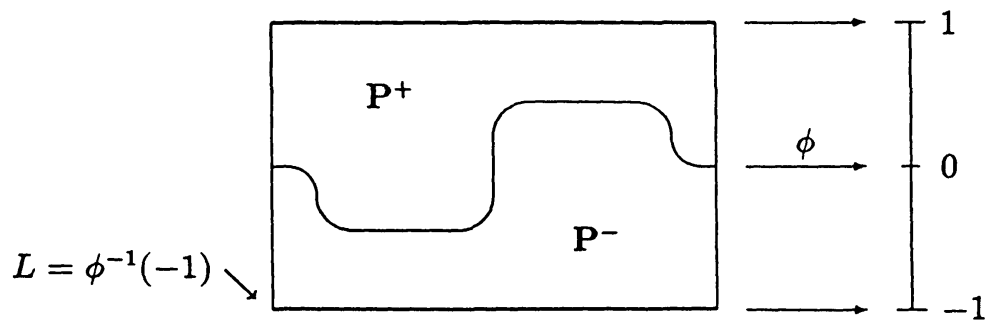

and so the cohomology $H^{p-1}(\mathbf{P}-L, \mathscr{O}(k))$ gives a representation of $J$. It is evident from the Laurent series expansion that the elementary states are precisely the $J$-finite vectors of this representation.

The elementary states are usually regarded, however, as cohomology classes in $H^{p-1}\left(\mathbf{P}^{+}, \mathscr{O}(k)\right)$ where they are referred to as elementary states based on $L$. This is legitimate because either by the Penrose transform [15] or by reasoning supplied in the previous section, the natural map

$$
H^{p-1}(\mathbf{P}-L, \mathscr{O}(k)) \rightarrow H^{p-1}\left(\mathbf{P}^{+}, \mathscr{O}(k)\right)
$$

is injective. Observe that $L$ can be any linearly embedded $\mathbf{P}_{q-1}$ inside $\mathbf{P}^{-}$. $\mathrm{U}(p, q)$ acts transitively on the set of possible choices and the stabilizer of $L$ is the maximal compact $\mathrm{U}(p) \times \mathrm{U}(q)$. Conversely, a choice of $\mathrm{U}(p) \times \mathrm{U}(q) \subset \mathrm{U}(p, q)$ stabilizes exactly one such $L$. Equivalently, $\mathrm{U}(p) \times \mathrm{U}(q)$ stabilizes $L^{\perp} \subset \mathbf{P}^{+}$, a linearly embedded $\mathbf{P}_{p-1}$. At this stage, however, it is not at all clear that the elementary states are the $\mathrm{U}(p) \times \mathrm{U}(q)$-finite vectors inside the $\mathrm{U}(p, q)$-module 
$H^{p-1}\left(\mathbf{P}^{+}, \mathscr{O}(k)\right)$. Though this is true, it requires further argument (supplied at the end of this paper).

Finally, by tensoring through with $\operatorname{det} \mathbf{T}$, note that the elementary states for

$$
H^{p-1}\left(\mathbf{P}^{+}, \mathscr{O}(a \mid b, \ldots, b)\right)
$$

are grouped into the representations

$$
(b+1, \ldots, b+1, b+1+d-p \mid a-d, b, \ldots, b)
$$

of $\mathrm{U}(p) \times \mathrm{U}(q)$ for $d \geq \max (p, a-b)$. When viewed inside $H^{p-1}(\mathbf{P}-L, \mathscr{O}(a \mid b, \ldots, b))$, the lower left-hand block of $J$ acts as lowering operators from elementary states of "level" $d$ to those of level $d-1$. This can be seen explicitly on Čech cocycles where the Lie algebra of $J$ acts as differential operators $[5,6]$.

4. The ladder representations. In this section we place the cohomologies that we have been studying in the context of general representation theory. This is slightly jumping the gun since, as remarked above, we do not yet know that the elementary states are the $K$-finite vectors in these various cohomologies. Here $K$ denotes the maximal compact subgroup

$$
K=\mathrm{U}(p) \times \mathrm{U}(q) \subset \mathrm{U}(p, q)
$$

and it is a standard technique in the study of representations of reductive Lie groups to first restrict to such a $K$ and see how the $K$-finite vectors of the representation decompose into irreducibles. Thus, for the purposes of this section we assume to know that the elementary states are the $K$-finite vectors (as proved in $\S 7$ ).

As shown in the previous section, the $K$-types of

$$
H^{p-1}\left(\mathbf{P}^{+}, \mathscr{O}(\lambda)\right) \text { or } H^{p-1}\left(\overline{\mathbf{P}^{+}}, \mathscr{O}(\lambda)\right)
$$

are especially simple. There is a highest $K$-type (where $d=$ $\max (p, a-b))$ and then a single ray of $K$-types (as $d$ increases) all occurring with multiplicity one. It is for this reason that these representations are known to physicists as the ladder representations.

The $K$-finite parts of these representations agree-they constitute the same Harish Chandra module. A Fréchet representation with a particular Harish Chandra module as $K$-finite part is called a globalization of this module and it is shown in $[10]$ that $H^{p-1}\left(\mathbf{P}^{+}, \mathscr{O}(\lambda)\right)$ is precisely the hyperfunction or maximal globalization [42]. This is also a special case of a very general result of Schmid and Wolf $[43,44]$ which uses geometric quantization to produce Fréchet representations 
which are the maximal globalizations of Zuckerman's derived functor modules. (We are grateful to the referee for drawing our attention to this result.) More interesting, however, is the fact that these modules are unitarizable i.e. admit a (necessarily unique) Hilbert space globalization. This is proved in $\S 6$ below but is already well-known. We now review some of the relevant literature and explain why the "twistor" method is quite different from the usual approaches.

As mentioned in the introduction, the case of $\operatorname{SU}(2,2)$ is especially interesting from the physical point of view since there are the 2-1 covers

$$
\mathrm{SU}(2,2) \rightarrow \mathrm{O}_{+}^{\uparrow}(2,4) \rightarrow \mathrm{C}_{+}^{\uparrow}(1,3)
$$

where $C_{+}^{\uparrow}(1,3)$ is the identity component of the group of conformal transformations of compactified Minkowski space. In this case the ladder representations occur as the spaces of massless fields of various helicities on compactified Minkowski space and physicists have long known how to unitarize them (though usually regarded only as representations of the Poincare group, a subgroup of the conformal group). Incidently, that is why the work state is employed-from the language of quantum mechanics. One can Fourier analyze these fields and directly verify invariance of the physically motivated scalar product. This approach is detailed in [28]. The calculations required are fairly severe. This method generalizes immediately to the case of $\mathrm{O}_{+}^{\uparrow}(2, k)$ and, with more effort, to the case of $\mathrm{SU}(p, q)$ (as in [30]).

Since then, much progress has been made in unitarizing these and other modules. The simple pattern of $K$-types shows that the ladder representations are examples of highest weight modules i.e. they are generated by lowering a single weight vector. It is now known precisely which such modules are unitarizable $[18,29]$ and there are several methods of proving unitarity (e.g. $[47,1])$. These methods are usually algebraic.

More closely related to the twistor method, however, is the construction of representations on $L^{2}$-cohomology. This was very successful for the discrete series [41, 4] and this approach has since been adapted [38] to deal with certain singular representations (of which the ladder representations are examples). However, we should point out that, although there is always a mapping from $L^{2}$-cohomology to ordinary Dolbeault cohomology, they are not usually well-related.

There is always a difficulty in establishing the unitary structure. For $L^{2}$-cohomology this difficulty lies in showing that the answer is nontrivial either from Harish Chandra's original proof of the existence of 
the discrete series as in [41] or from an $L^{2}$-index theorem as in [4]. What is clear in this approach, however, is the existence and invariance of the Hilbert space inner product. These difficulties are compounded for the singular representations [38] when the inner product is not so clear. For realizations such as those on massless fields the existence of the Hilbert space is straightforward but work is needed to check invariance of the inner product under the action of the group. Indeed, sometimes it is not so clear that the group acts at all-this involves checking that the field equations are conformally invariant $[24,8,17]$.

With the twistor approach, however, the existence of the representation (without the unitary structure) is elementary since Dolbeault cohomology is well-understood (cf. Schmid's original approach to the discrete series [40]). The construction of an invariant Hermitian form requires some work but is straightforward once the twistor transform used in its construction is under control. The place where the twistor construction has its difficulties is in showing that the scalar product is positive definite. This is where the elementary states are so useful (as in $\S \S 6$ and 7 below).

The twistor construction of the scalar product for the case of massless fields first appears in [35, pp. 277-278] where it is couched in the language of twistor diagrams. It is obtained by transferring the spacetime definition over to twistor space. At this time the Penrose transform was in an early stage of development. Penrose had not yet recast it in terms of cohomology. Some cohomological implications of the twistor construction were worked out in $[20,21,13]$. The work of Rawnsley, Schmid, and Wolf [38] was also inspired in part by Penrose's results though their conclusions are rather different than ours.

Finally, we should note that the invariance of the massless field equations and their relatives under the group of conformal transformations is a special case of a much stronger invariance, namely invariance under local conformal rescalings of the metric. Further discussion on this aspect can be found in $[8,17]$.

\section{Proof of density.}

Theorem 3. For a fixed $L \subset \mathbf{P}^{-}$the elementary states based on $L$ are dense in $H^{p-1}\left(\mathbf{P}^{+}, \mathscr{O}(\lambda)\right)$.

Proof. By the Hahn-Banach theorem and Theorem 2, we must show that if $F \in H^{q-1}\left(\overline{\mathbf{P}^{-}}, \mathscr{O}(\tilde{\lambda})\right)$ and $e \cdot F=0$ for all elementary states $e$, then $F=0$. Suppose $\tilde{\lambda}=(a \mid b, \ldots, b)$. According to $\S 1$, the 
simplest $(d=\max (p, a-b))$ elementary states based on $L$ comprise a finite dimensional representation $R_{0}$ of $\mathrm{U}(p) \times \mathrm{U}(q)$, namely:

$$
\begin{array}{ll}
(b+1, \ldots, b+1, b+1 \mid a-p, b, \ldots, b) & \text { if } p \geq a-b \\
(b+1, \ldots, b+1, a+1-p \mid b, b, \ldots, b) & \text { if } p \leq a-b .
\end{array}
$$

Taking the dot product with these states gives

$$
R_{0} \otimes H^{q-1}\left(\overline{\mathbf{P}^{-}}, \mathscr{O}(\tilde{\lambda})\right) \rightarrow \mathbf{C}
$$

or, equivalently, a natural map

$$
H^{q-1}\left(\overline{\mathbf{P}^{-}}, \mathscr{O}(\tilde{\lambda})\right) \rightarrow R_{0}^{*} .
$$

The $(q-1)$-plane $L$ in $\mathbf{P}^{-}$may be regarded as a point in the Grassmannian $\operatorname{Gr}_{q}(\mathbf{T})$. Choosing different $L$ in $\mathbf{P}^{-}$sweeps out an open subset $\mathbf{M}^{-}$of $\mathrm{Gr}_{q}(\mathbf{T})$. The generalized Penrose transform [15] interprets $H^{q-1}\left(\overline{\mathbf{P}^{-}}, \mathscr{O}(\tilde{\lambda})\right)$ as holomorphic solutions of certain linear holomorphic differential equations on $\overline{\mathbf{M}^{-}}$. Details of this transform are explained in $[14,15,6]$ and shall be omitted here. It is shown in [14] that the map $H^{q-1}\left(\overline{\mathbf{P}^{-}}, \mathscr{O}(\tilde{\lambda})\right) \rightarrow R_{0}^{*}$ is exactly the evaluation of the Penrose transform at $L \in \mathbf{M}^{-}$. Similarly, the elementary states in

$$
\begin{array}{ll}
R_{1} \equiv(b+1, \ldots, b+1, b+2 \mid a-p-1, b, \ldots, b) & \text { if } p \geq a-b, \\
R_{1} \equiv(b+1, \ldots, b+1, a+2 p-\mid b-1, b, \ldots, b) & \text { if } p \leq a-b,
\end{array}
$$

define a homomorphism

$$
H^{q-1}\left(\overline{\mathbf{P}^{-}}, \mathscr{O}(\tilde{\lambda})\right) \rightarrow R_{1}^{*}
$$

which coincides with the derivative of the Penrose transform at $L$ (see [13] for the case $p=q=2$ ). Higher elementary states give higher derivatives. Thus $e \cdot F=0$ for all $e$ based on $L$ if and only if the Penrose transform of $F$ vanishes together with all its derivatives at $L \in \mathbf{M}^{-}$. By analyticity, this means that the Penrose transform of $F$ is identically zero and hence $F$ itself vanishes, as required.

REMARKS. 1. A similar proof applies for the elementary states in $H^{q-1}\left(\overline{\mathbf{P}^{+}}, \mathscr{O}(\lambda)\right)$.

2. The method of proof shows slightly more than just density and this will be used in the next section.

6. The scalar product. The main result of [14] (also proved in [6]) is that there is a canonical isomorphism of topological vector spaces, the twistor transform

$$
\mathscr{T}: H^{p-1}\left(\overline{\mathbf{P}^{+}}, \mathscr{O}(\lambda)\right) \rightarrow H^{q-1}\left(\overline{\mathbf{P}^{*-}}, \mathscr{O}(\tilde{\lambda})\right)
$$


where, if $\lambda=(a \mid b, \ldots, b)$ then $\tilde{\lambda}=(-a+n-1 \mid-b-1, \ldots,-b-1)$. It is obtained through Penrose transforming these two cohomologies and should be regarded as a complex version of the Fourier transform, the Penrose transform being a complex version of the Radon transform. In particular, this isomorphism is $U(p, q)$-equivariant. The Hermitian form $\Phi$ on $\mathbf{T}$ induces a conjugate linear isomorphism

$$
H^{q-1}\left(\overline{\mathbf{P}^{*-}}, \mathscr{O}(\tilde{\lambda})\right) \stackrel{\simeq}{\rightrightarrows} H^{q-1}\left(\overline{\mathbf{P}^{-}}, \mathscr{O}(\tilde{\lambda})\right)
$$

by $\bar{f}(Z)=\overline{f(\bar{Z})}$ on Čech cocycles. Combining this with the $\operatorname{dot}$ product gives an Hermitian product, the scalar product, $\langle\mid\rangle$, on $H^{p-1}\left(\overline{\mathbf{P}^{+}}, \mathscr{O}(\lambda)\right)$ by

$$
\langle\omega \mid \mu\rangle \equiv \omega \cdot \overline{\mathscr{T}} \mu \text {. }
$$

This product is manifestly $\mathrm{U}(p, q)$-invariant and we shall now show that it is positive definite.

By direct calculation $[35,13,14]$, the elementary states in $H^{p-1}\left(\overline{\mathbf{P}^{+}}, \mathscr{O}(\lambda)\right)$ based on $L \subset \mathbf{P}^{-}$are mapped isomorphically by the twistor transform to the elementary states in $H^{q-1}\left(\overline{\mathbf{P}^{*-}}, \mathscr{O}(\tilde{\lambda})\right)$ based on $\overline{L^{\perp}} \subset \mathbf{P}^{*+}$. It is then straightforward to verify that these finite dimensional representations of $\mathrm{U}(p) \times \mathrm{U}(q)$ are mutually orthogonal for $\langle\mid\rangle$ and that $\langle\mid\rangle$ is positive definite on each. It is also clear that $\langle\mid\rangle$ is continuous for the natural topology on $H^{p-1}\left(\overline{\mathbf{P}^{+}} \mathscr{O}(\lambda)\right)$. Thus, from the density shown in $\S 3$, it follows that $\langle\mid\rangle$ is non-negative definite. However, it is actually positive definite for suppose that $\langle\mu \mid \mu\rangle=0$. Then for any $t \in \mathrm{C}$ and $e$ an elementary state based on $L$

$$
0 \leq\langle t \mu+e \mid t \mu+e\rangle=2 \operatorname{Re}(t\langle\mu \mid e\rangle)+\langle e \mid e\rangle .
$$

Thus, $\langle\mu \mid e\rangle=0$ for all elementary states based on $L$. Since the twistor transform preserves elementary states, this is equivalent to saying that $\mu \cdot f=0$ for all elementary states $f$ based on $L^{\perp}$. According to the proof of the previous theorem, this implies that $\mu=0$ as required.

7. The $K$-finite states are elementary. We conclude with the following remarks:

1. The $L^{2}$-completion of $H^{p-1}\left(\overline{\mathbf{P}^{+}}, \mathscr{O}(\lambda)\right)$ with respect to $\langle\mid\rangle$ is called the space of normalizable states and lies between $H^{p-1}\left(\overline{\mathbf{P}^{+}}, \mathscr{O}(\lambda)\right)$ and $H^{q-1}\left(\mathbf{P}^{+}, \mathscr{O}(\lambda)\right)$ as a cohomological generalization of $L^{2}$-functions between real analytic functions and hyperfunctions.

2. Using $\langle\mid\rangle$ allows one to expand an element of $H^{p-1}\left(\overline{\mathbf{P}^{+}}, \mathscr{O}(\lambda)\right)$ as a series in elementary states. This is a stronger statement than 
just density. When $p=1$ this reduces to expanding a function as a power series about a chosen point (whereas density is merely the Runge approximation theorem).

3. By viewing elements of $H^{p-1}\left(\overline{\mathbf{P}^{+}}, \mathscr{O}(\lambda)\right)$ as such series, one can directly compare $H^{p-1}\left(\overline{\mathbf{P}^{+}}, \mathscr{O}(\lambda)\right)$ and $H^{p-1}(\mathbf{P}-L, \mathscr{O}(\lambda))$. A similar expansion is possible for elements of $H^{p-1}\left(\mathbf{P}^{+}, \mathscr{O}(\lambda)\right)$ since one can calculate coefficients by pairing with the elementary states in $H^{q-1}\left(\mathbf{P}-L^{\perp}, \mathscr{O}(\tilde{\lambda})\right)$. Thus, all of these cohomologies may be regarded as Laurent series as in $\S 3$. They differ merely because their notions of convergence are different. In all cases, however, it is now clear that the $\mathrm{U}(p) \times \mathrm{U}(q)$-finite vectors are those with finite Laurent expansions. In other words, the elementary states are precisely the $\mathrm{U}(p) \times \mathrm{U}(q)$-finite vectors.

4. Had we known this already, the density of Theorem 3 would have followed from general arguments-the $K$-finite vectors in a Fréchet module are always dense [46].

5. It is easy to see that $\mathrm{U}(p, q)$ acts irreducibly on the elementary states. Now that we know that these coincide with the $\mathrm{U}(p) \times \mathrm{U}(q)$ finite vectors, it follows that $H^{p-1}\left(\mathbf{P}^{+}, \mathscr{O}(\lambda)\right)$ or the Hilbert space completion of $H^{p-1}\left(\overline{\mathbf{P}^{+}}, \mathscr{O}(\lambda)\right)$ are irreducible $\mathrm{U}(p, q)$-modules.

\section{REFERENCES}

[1] J. Adams, Unitary highest weight modules, Adv. in Math., 63 (1987), 113-137.

[2] A. Andreotti and $\mathrm{H}$. Grauert, Théorèmes de finitude pour cohomologie des espaces complexes, Bull. Soc. Math. France, 90 (1962), 193-259.

[3] A. Andreotti and C. D. Hill, E. E. Levi convexity and the Hans Lewy problem I, II, Ann. Scuola Norm. Sup. Pisa, 26 (1972), 325-363 and 747-806.

[4] M. F. Atiyah and W. Schmid, A geometric construction of the discrete series for semisimple Lie groups, Inv. Math., 42 (1977), 1-62.

[5] R. J. Baston, Local cohomology, elementary states, and evaluation, Twistor Newsletter, 22 (1986), 8-13 (reprinted in "Further Advances in Twistor Theory", (eds. L. J. Mason and L. P. Hughston), pp. 144-151, Pitman Research Notes in Math., 231, Longman, Harlow 1990).

[6] R. J. Baston and M. G. Eastwood, The Penrose Transform: its Interaction with Representation Theory, Oxford University Press, 1989.

[7] R. Bott, Homogeneous vector bundles, Ann. Math., 66 (1957), 203-248.

[8] T. P. Branson, Group representations arising from Lorentz conformal geometry, J. Funct. Anal., 74 (1987), 199-291.

[9] H. Cartan and J.-P. Serre, Un théorème de finitude concernant les variétés analytiques compactes, C. R. Acad. Sci. Paris, 237 (1953), 128-130.

[10] E. G. Dunne, Hyperfunctions in representation theory and mathematical physics in "Integral Geometry", (eds. R. L. Bryant et al.), pp. 51-65, Contemp. Math., vol. 63, Amer. Math. Soc., Providence, RI, 1987. 
[11] M. G. Eastwood and L. P. Hughston, Massless fields based on a line, in [27], 101-109.

[12] M. G. Eastwood, R. Penrose, and R. O. Wells, Jr., Cohomology and massless fields, Comm. Math. Phys., 78 (1981), 305-351.

[13] M. G. Eastwood and M. L. Ginsberg, Duality in twistor theory, Duke Math. J., 48 (1981), 177-196.

[14] M. G. Eastwood, The generalized twistor transform and unitary representations of $\mathrm{SU}(p, q)$, preprint.

[15] _ The generalized Penrose-Ward transform, Math. Proc. Camb. Phil. Soc., 97 (1985), 165-187.

[16] _ A duality for homogeneous bundles on twistor space, J. London Math. Soc., 31 (1985), 349-356.

[17] M. G. Eastwood and J. W. Rice, Conformally invariant differential operators on Minkowski space and their curved analogues, Comm. Math. Phys., 109 (1987), 207-228.

[18] T. J. Enright, R. Howe, and N. R. Wallach, A classification of unitary highest weight modules, in "Representation Theory of Reductive Lie Groups", (ed. P. C. Trombi), pp. 268-407, Birkhäuser, Basel/Boston/Stuttgart, 1983.

[19] M. Fierz, Über den Drehimpuls von Teilchen mit Ruhemasse null und beliebigen Spin, Helv. Phys. Acta, 13 (1940), 45-60.

[20] M. L. Ginsberg, A cohomological scalar product construction, in [27], 293-300.

[21] _ A Cohomological Approach to Scattering Theory, D. Phil. thesis, Oxford University, 1980.

[22] R. Godement, Topologie Algébrique et Théorie des Faisceaux, Hermann, Paris, 1964.

[23] E. Grgin, A Global Technique for the Study of Spinor Fields, Ph.D. thesis, Syracuse University, 1966.

[24] L. Gross, Norm invariance of mass-zero equations under the conformal group, J. Math. Phys., 5 (1963), 687-695.

[25] C. D. Hill, The Cauchy problem for $\bar{\partial}$, in "Partial Differential Equations", Proc. Sympos. Pure Math., vol. 23, Amer. Math. Soc., Providence, RI, 1973, pp. $135-143$.

[26] A. P. Hodges, Twistor diagrams, Physica, 114A (1982), 157-175.

[27] L. P. Hughston and R. S. Ward (eds.), Advances in Twistor Theory, Research Notes in Math., 37, Pitman, San Francisco/London/Melbourne, 1979.

[28] H. P. Jakobsen and M. Vergne, Wave and Dirac operators and representations of the conformal group, J. Funct. Anal., 24 (1977), 52-106.

[29] H. P. Jakobsen, Hermitian symmetric spaces and their unitary highest weight modules, J. Funct. Anal., 52 (1983), 385-412.

[30] M. Kashiwara and M. Vergne, On the Segal-Shale-Weil representations and harmonic polynomials, Inv. Math., 44 (1978), 1-47.

[31] H. B. Laufer, On sheaf cohomology and envelopes of holomorphy, Ann. Math., 84 (1966), 102-118.

[32] _ - On Serre duality and envelopes of holomorphy, Trans. Amer. Math. Soc., 128 (1967), 414-436.

[33] J. A. McLennan, Jr., Conformal invariance and conservation laws for relativistic wave equations for zero rest mass, Nuovo Cimento, 3 (1956), 1360-1379.

[34] R. Penrose, Twistor algebra, J. Math. Phys., 8 (1967), 345-366.

[35] R. Penrose and M.A.H. MacCallum, Twistor theory: an approach to the quantisation of fields and space-time, Phys. Rep., 6C (1972), 241-316.

[36] R. Penrose, Twistor theory, its aims and achievements, in "Quantum Gravity: an Oxford Symposium", pp. 268-407, Clarendon Press, Oxford, 1975. 
[37] R. Penrose and W. Rindler, Spinors and Space-time, Vol. 2, Cambridge University Press, 1986.

[38] J. Rawnsley, W. Schmid, and J. A. Wolf, Singular unitary representations and indefinite harmonic theory, J. Funct. Anal., 51 (1983), 1-114.

[39] G. Scheja, Riemannsche Hebbarkeitssätze für Cohomologieklassen, Math. Ann., 144 (1961), 345-360.

[40] W. Schmid, Homogeneous complex manifolds and representations of semisimple Lie groups, Ph.D. thesis, University of California-Berkeley, 1967.

[41] __, $L^{2}$-cohomology and the discrete series, Ann. Math., 103 (1976), 375-394.

[42] __ Boundary value problems for group invariant differential equations, in "Proceedings of the Cartan Symposium, Lyon 1984", pp. 311-322, Astérisque, 1985.

[43] W. Schmid and J. A. Wolf, Globalizations of Harish Chandra modules, Bull. Amer. Math. Soc., 17 (1987), 117-120.

[44] W. Schmid and J. A. Wolf, Geometric quantization and derived functor modules for semisimple Lie groups, J. Funct. Anal., 90 (1990), 48-112.

[45] J.-P. Serre, Un théorème de dualité, Comm. Math. Helv., 29 (1955), 9-26.

[46] G. Warner, Harmonic Analysis on Semi-simple Lie Groups I, Springer, Berlin/ Heidelberg/New York, 1972.

[47] R. Zierau, Geometric construction of certain highest weight modules, Proc. Amer. Math. Soc., 95 (1985), 631-635.

Received August 22, 1986 and in revised form April 24, 1991.

UNIVERSITY OF ADELAIDE

G.P.O. BoX 498

Adelaide, South Australia 5001

AND

Morgan Guaranty Ltd.

P.O. BOX 124

30 THROGMORTON StREeT

LONDON EC2N 2NT, ENGLAND 



\section{PACIFIC JOURNAL OF MATHEMATICS EDITORS}

\author{
V. S. VARADARAJAN \\ (Managing Editor) \\ University of California \\ Los Angeles, CA 90024-1555-05 \\ Herbert Clemens \\ University of Utah \\ Salt Lake City, UT 84112 \\ THOMAS ENRIGHT \\ University of California, San Diego \\ La Jolla, CA 92093
}

Nicholas ERcolani

University of Arizona

Tucson, AZ 85721

R. FINN

Stanford University

Stanford, CA 94305

VAUGHAN F. R. JONES

University of California

Berkeley, CA 94720

STEVEN KeRCKHOFF

Stanford University

Stanford, CA 94305

\section{C. MOORE \\ University of California \\ Berkeley, CA 94720}

MARTIN SChaRLEMANN

University of California

Santa Barbara, CA 93106

HAROLd STARK

University of California, San Diego

La Jolla, CA 92093

\section{ASSOCIATE EDITORS}

\begin{tabular}{|c|c|c|c|c|}
\hline ARENS & $\begin{array}{l}\text { E. F. BECKENBACH } \\
(1906-1982)\end{array}$ & NeumanN & $\begin{array}{l}\text { F. Wolf } \\
(1904-1989)\end{array}$ & K. Yoshida \\
\hline \multicolumn{5}{|c|}{ SUPPORTING INSTITUTIONS } \\
\hline \multirow{2}{*}{\multicolumn{2}{|c|}{$\begin{array}{l}\text { IVERSITY OF ARIZONA } \\
\text { IIVERSITY OF BRITISH COLUMBIA }\end{array}$}} & UNIVERS & Y OF OREGON & \\
\hline & & UNIVERS & OF SOUTHER & IIA \\
\hline \multicolumn{2}{|c|}{ LIFORNIA INSTITUTE OF TECHNOLOGY } & STANFO & UNIVER & \\
\hline \multirow{2}{*}{\multicolumn{2}{|c|}{$\begin{array}{l}\text { IVERSITY OF CALIFORNIA } \\
\text { ONTANA STATE UNIVERSITY }\end{array}$}} & UNIVERS & OF HAWAII & \\
\hline \multirow{2}{*}{\multicolumn{2}{|c|}{$\begin{array}{l}\text { ONTANA STATE UNIVERSITY } \\
\text { IIVERSITY OF NEVADA, RENO }\end{array}$}} & UNIVERS & YF TOKYO & \\
\hline & & UNIVERS & I OF UTAH & \\
\hline \multirow{2}{*}{\multicolumn{2}{|c|}{$\begin{array}{l}\text { W MEXICO STATE UNIVERSITY } \\
\text { EGON STATE UNIVERSITY }\end{array}$}} & WASHING & N STATE UNI & SITY \\
\hline & & UNIVERS & NASI & \\
\hline
\end{tabular}

The Supporting Institutions listed above contribute to the cost of publication of this Journal, but they are not owners or publishers and have no responsibility for its content or policies.

Mathematical papers intended for publication in the Pacific Journal of Mathematics should be in typed form or offset-reproduced (not dittoed), double spaced with large margins. Please do not use built up fractions in the text of the manuscript. However, you may use them in the displayed equations. Underline Greek letters in red, German in green, and script in blue. The first paragraph must be capable of being used separately as a synopsis of the entire paper. In particular it should contain no bibliographic references. Please propose a heading for the odd numbered pages of less than 35 characters. Manuscripts, in triplicate, may be sent to any one of the editors. Please classify according to the 1991 Mathematics Subject Classification scheme which can be found in the December index volumes of Mathematical Reviews. Supply name and address of author to whom proofs should be sent. All other communications should be addressed to the managing editor, or Elaine Barth, University of California, Los Angeles, California 90024-1555-05.

There are page-charges associated with articles appearing in the Pacific Journal of Mathematics. These charges are expected to be paid by the author's University, Government Agency or Company. If the author or authors do not have access to such Institutional support these charges are waived. Single authors will receive 50 free reprints; joint authors will receive a total of 100 free reprints. Additional copies may be obtained at cost in multiples of 50 .

The Pacific Journal of Mathematics (ISSN 0030-8730) is published monthly except for July and August. Regular subscription rate: $\$ 190.00$ a year (10 issues). Special rate: $\$ 95.00$ a year to individual members of supporting institutions.

Subscriptions, orders for numbers issued in the last three calendar years, and changes of address should be sent to Pacific Journal of Mathematics, P.O. Box 969, Carmel Valley, CA 93924, U.S.A. Old back numbers obtainable from Kraus Periodicals Co., Route 100, Millwood, NY 10546.

The Pacific Journal of Mathematics at P.O. Box 969, Carmel Valley, CA 93924 (ISSN 0030-8730) is published monthly except for July and August. Second-class postage paid at Carmel Valley, Californiä 93924, and additional mailing offices. Postmaster: send address changes to Pacific Journal of Mathematics, P.O. Box 969, Carmel Valley, CA 93924.

\section{PUBLISHED BY PACIFIC JOURNAL OF MATHEMATICS, A NON-PROFIT CORPORATION}




\section{Pacific Journal of Mathematics}

\section{Vol. 151, No. $2 \quad$ December, 1991}

Michael G. Eastwood and A. M. Pilato, On the density of twistor

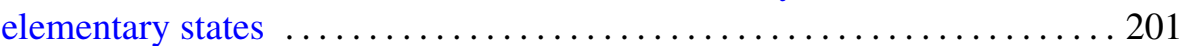

Brian E. Forrest, Arens regularity and discrete groups $\ldots \ldots \ldots \ldots \ldots \ldots 217$

Yu Li Fu, On Lipschitz stability for F.D.E ..................... 229

Douglas Austin Hensley, The largest digit in the continued fraction expansion of a rational number $\ldots \ldots \ldots \ldots \ldots \ldots \ldots \ldots \ldots \ldots \ldots . \ldots 237$

Uwe Kaiser, Link homotopy in $\mathbb{R}^{3}$ and $S^{3}$

Ronald Leslie Lipsman, The Penney-Fujiwara Plancherel formula for abelian symmetric spaces and completely solvable homogeneous

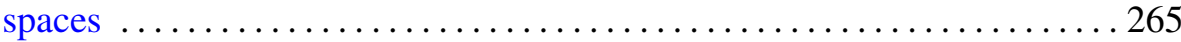

Florin G. Radulescu, Singularity of the radial subalgebra of $\mathscr{L}\left(F_{N}\right)$ and the

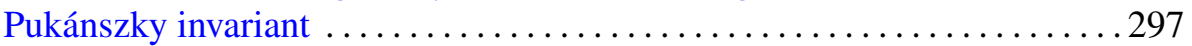

Albert Jeu-Liang Sheu, The structure of twisted SU(3) groups $\ldots . \ldots \ldots . \ldots 307$

Morwen Thistlethwaite, On the algebraic part of an alternating link . . . . . 317

Thomas (Toma) V. Tonev, Multi-tuple hulls .................. 335

Arno van den Essen, A note on Meisters and Olech's proof of the global asymptotic stability Jacobian conjecture ..................... 351

Hendrik J. van Maldeghem, A characterization of the finite Moufang hexagons by generalized homologies

Bun Wong, A note on homotopy complex surfaces with negative tangent bundles

Chung-Tao Yang, Any Blaschke manifold of the homotopy type of $\mathbf{C} P^{n}$ has the right volume 